\title{
Impactos da pandemia da Covid-19 em profissionais da Psicologia
}

\author{
Impacts of the Covid-19 pandemic on Psychology professionals \\ Impactos de la pandemia del Covid-19 en lós profesionales de lá Psicología
}

Recebido: 22/03/2021 | Revisado: 30/03/2021 | Aceito: 02/04/2021 | Publicado: 14/04/2021

\author{
Nathália Bonugli Caurin \\ ORCID: https://orcid.org/0000-0002-1788-1823 \\ Universidade São Francisco, Brasil \\ E-mail: nathaliacaurin@gmail.com \\ Antonio Renan Santana \\ ORCID: https://orcid.org/0000-0003-3408-7995 \\ Universidade Federal do Ceará, Brasil \\ E-mail: antoniorenan@outlook.com \\ Antônio Breno Gomes de Negreiros \\ ORCID: https://orcid.org/0000-0001-6553-5258 \\ Universidade Federal do Ceará, Brasil \\ E-mail: brenonegreiros@alu.com.br \\ Mirlly de Souza Ferreira \\ ORCID: https://orcid.org/0000-0002-9451-2415 \\ Universidade Federal do Ceará, Brasil \\ E-mail: mirllydesouzaf@gmail.com \\ Victória Maria Freitas Pedrosa \\ ORCID: https://orcid.org/0000-0002-7467-6973 \\ Universidade Federal do Ceará, Brasil \\ E-mail: vicmaripedrosa16@gmail.com \\ Jocélia Medeiros Ximenes \\ ORCID: https://orcid.org/0000-0001-9850-4423 \\ Universidade Federal do Ceará, Brasil \\ E-mail: jocelia_mx@yahoo.com.br \\ Luiz Wescley Fontenele Moura \\ ORCID: https://orcid.org/0000-0001-6198-6385 \\ Faculdade IEDucare, Brasil \\ E-mail: luizwescley@hotmail.com \\ Jeferson da Silva Rodrigues \\ ORCID: https://orcid.org/0000-0002-3112-9478 \\ Faculdade IEDucare, Brasil \\ E-mail: jeffersonrdo2000@gmail.com \\ Juliana Maria da Silva Trajano \\ ORCID: https://orcid.org/0000-0001-7518-7349 \\ Faculdade IEDucare, Brasil \\ E-mail: julianatrajanopsi@gmail.com \\ André Sousa Rocha \\ ORCID: https://orcid.org/0000-0002-0185-9699 \\ Universidade São Francisco, Brasil \\ E-mail: andresousarocha9@gmail.com
}

\begin{abstract}
Resumo
A pandemia ocasionada pelo coronavírus Sars-CoV-2 inseriu os profissionais da área da saúde em constante desafio. Assim, com o profissional da psicologia inserido nesse contexto, torna-se importante compreender quais os impactos provenientes deste cenário. Em vista da escassez de pesquisas sobre o tema até o momento, objetivou-se verificar quais os impactos que a pandemia ocasionou para os psicólogos que estão atuando na linha de frente no âmbito hospitalar e da clínica. Para tanto, utilizou-se de uma revisão integrativa da literatura, realizada a partir da busca por publicações científicas indexadas na plataforma Google Scholar, no periódico da Coordenação de Aperfeiçoamento de Pessoal de Nível Superior, na Scientific Eletronic Librabry Online e nas bases de dados da Literatura Latino-Americano e do Caribe em Ciências da Saúde e Portal de Periódicos Eletrônicos de Psicologia, ambos inseridos na Biblioteca Virtual em Saúde. Resultou-se na seleção de quatro artigos para essa revisão. Diante dos achados, percebeu-se o desenvolvimento em profissionais de psicologia sintomas relacionados ao estresse, ansiedade, depressão e abuso de substâncias. Ademais, o psicólogo encontra dificuldades com relação ao fluxo, emergência e contato com os pacientes, de modo a ter também que se adaptar rapidamente a realidade dos atendimentos on-line para intervenção psicológica. Essa pesquisa permitiu refletir sobre a necessidade do olhar cuidadoso com psicólogos no que tange a problemas traumáticos e sociais gerados pela pandemia. Além disso, constatou-se a necessidade de novos estudos para ampliar o panorama dos impactos para além dos psicólogos que atuam na atenção terciária e clínica.
\end{abstract}


Palavras-chave: Atenção terciária à saúde; Atendimento on-line; Esgotamento emocional, Coronavírus.

\begin{abstract}
The pandemic caused by the Sars-CoV-2 coronavirus has placed health professionals in constant challenge. Thus, with the psychology professional inserted in this context, it is important to understand the impacts arising from this scenario. In view of the scarcity of research on the subject so far, the objective was to verify what impacts the pandemic has caused for psychologists who are working on the front lines in the hospital and clinic. To this end, an integrative literature review was carried out, based on the search for scientific publications indexed on the Google Scholar platform, in the journal of the Coordination for the Improvement of Higher Education Personnel, in the Scientific Eletronic Librabry Online and in the databases of the Latin American and Caribbean Literature on Health Sciences and Portal of Electronic Journals on Psychology, both inserted in the Virtual Health Library. This resulted in the selection of four articles for this review. In view of the findings, the development of symptoms related to stress, anxiety, depression and substance abuse was noticed in psychology professionals. In addition, the psychologist finds difficulties in relation to flow, emergency and contact with patients, in order to also have to quickly adapt to the reality of online care for psychological intervention. This research allowed us to reflect on the need for careful observation with psychologists regarding traumatic and social problems generated by the pandemic. In addition, there was a need for further studies to expand the panorama of impacts beyond the psychologists who work in tertiary and clinical care.
\end{abstract}

Keywords: Tertiary health care; Online service; Emotional exhaustion, Coronavirus.

\title{
Resumen
}

La pandemia causada por el coronavirus Sars-CoV-2 ha puesto a los profesionales de la salud en un desafío constante. Así, con el profesional de la psicología insertado en este contexto, es importante comprender los impactos que se derivan de este escenario. Ante la escasez de investigaciones sobre el tema hasta el momento, el objetivo era verificar qué impactos ha causado la pandemia a los psicólogos que se encuentran trabajando en primera línea en el hospital y la clínica. Para ello, se realizó una revisión integradora de la literatura, a partir de la búsqueda de publicaciones científicas indexadas en la plataforma Google Scholar, en la revista de la Coordinación para el Perfeccionamiento del Personal de Educación Superior, en la Scientific Eletronic Librabry Online y en las bases de datos. de la Literatura Latinoamericana y del Caribe en Ciencias de la Salud y el Portal de Revistas Electrónicas de Psicología, ambos insertados en la Biblioteca Virtual en Salud, lo que resultó en la selección de cuatro artículos para esta revisión. A la vista de los hallazgos, se notó el desarrollo de síntomas relacionados con el estrés, la ansiedad, la depresión y el abuso de sustancias en los profesionales de la psicología. Además, el psicólogo encuentra dificultades en relación al flujo, la emergencia y el contacto con los pacientes, para tener que adaptarse rápidamente a la realidad de la atención online para la intervención psicológica. Esta investigación nos permitió reflexionar sobre la necesidad de una mirada atenta con los psicólogos sobre los problemas traumáticos y sociales generados por la pandemia. Además, era necesario realizar más estudios para ampliar el panorama de impactos más allá de los psicólogos que trabajan en la atención terciaria y clínica.

Palabras clave: Atención terciaria de salud; Servicio en línea; Agotamiento emocional, Coronavirus.

\section{Introdução}

Os meses finais de 2019 foram marcados pelo surgimento de um novo tipo de síndrome respiratória na China, causada pelo vírus SARS-CoV-2, que provocou a doença popularmente conhecida como COVID-19 ou Coronavírus. A alta transmissibilidade, a insuficiência respiratória leve ou aguda e o índice de letalidade são marcas dessa doença, além de quadros que envolvem febre, tosse seca, coriza, cansaço, perda de olfato e paladar, dentre outros. Dessa forma, devido ao alto poder de transmissão e infecção desse vírus, a Organização Mundial da Saúde (OMS) declarou a situação como Emergência de Saúde Pública de importância internacional em janeiro de 2020 e, posteriormente, caracterizou-ze a pandemia da COVID-19 (Brasil, 2020; Grincenkov, 2000; Prado, Peixoto, Silva e Scalia, 2020).

Para enfrentar a pandemia, medidas de promoção à saúde e prevenção de agravos foram adotadas pelas organizações em saúde, como a quarentena, isolamento e distanciamento social, proteção dos grupos mais vulneráveis além da utilização de máscaras, higienização frequente das mãos e uso de álcool em gel a 70\%. Contudo, tais medidas restritivas e de proteção podem desencadear repercussões negativas para a saúde mental, tais como, estresse crônico, ansiedade, tristeza, medo e insegurança (Brasil, 2020; Danzmann, Silva \& Guazina, 2020).

Nesse cenário, os profissionais de saúde ganharam maior protagonismo no combate à disseminação e tratamento da infecção pelo vírus, ao atuarem na linha de frente. No entanto, além de comporem um grupo de risco para o coronavírus, devido a maior exposição, também houve a necessidade de lidar com fatores relacionados à falta de equipamentos necessários, 
imprecisão dos protocolos de enfrentamento à doença e maior carga horária de trabalho (De Morais et al., 2021). A exaustão física, a pressão gerada pelo contexto pandêmico, o estresse psicológico, a preocupação, a ansiedade e o humor deprimido são igualmente observadas nesses profissionais, além de relatos de prejuízo na qualidade do sono, maior utilização de álcool, drogas e psicofármacos, medo da infecção ou de portar e transmitir o vírus, bem como quadros de transtornos de estresse pós-traumático e psicossomáticos (Grincenkov, 2020; Prado et al., 2020; Teixeira, Soares, Lisboa, Pinto, Andrade e Espiridião, 2020).

Vale destacar que o grupo composto por profissionais de saúde engloba todas as classes ocupacionais que são indispensáveis para atuação na linha de frente no combate à pandemia. Ou seja, enfermeiras/os, técnicas/os em enfermagem, médicas/os, psicólogas/os e fisioterapeutas são considerados os principais atores sociais envolvidos na pandemia. Além desses, diversos profissionais estão comprometidos e contribuem secundariamente no cuidado aos pacientes vitimados pelo coronavírus, tais como, motoristas, maqueiros, profissionais de serviços gerais, sepultadores e cremadores. Todas essas categorias profissionais enfrentam o risco de contaminação e estão sujeitas aos impactos na saúde física e mental que o contexto laboral e de pandemia ocasionam (Teixeira et al., 2020).

No contexto da psicologia hospitalar em atenção terciária a saúde, visa-se a redução do sofrimento gerado pela hospitalização, não apenas no âmbito da internação, mas também dos sentimentos e emoções eliciados pela patologia que motivou a hospitalização, e as consequências emocionais envolvidas. Ao trabalhar com a minimização do sofrimento, o psicólogo tem inúmeras possibilidades de atuação. Mas, para que o tratamento psicológico tenha êxito, é necessário que o profissional pondere as variáveis que permeiam o caso atendido. Essas variáveis podem ser psicológicas, emocionais ou físicas e de algum modo trazem prejuízo funcional na vida dos indivíduos (Angerami-Camon, 2001).

Adicionalmente, salienta-se que o profissional de psicologia, no ambiente hospitalar (atenção terciária a saúde em hospitais) e da clínica psicológica, está diretamente implicado na assistência aos pacientes, acompanhantes e equipes de saúde. Por esse motivo, há atuação no manejo do sofrimento psíquico, no apoio aos indivíduos afetados, bem como na orientação da administração de emoções, sentimentos, pensamentos e comportamentos (Danzmann; Silva; Guazina, 2020). Todavia, esse profissional também enfrenta desafios e precisa de suporte, visto que passou substancialmente a ser demandado em decorrência do período de pandemia e das afetações na saúde mental das pessoas e das equipes de saúde (De Moraes, Gomes, Machado, Daumas e Gomes, 2021).

Os principais objetos de trabalho do psicólogo hospitalar são o sofrimento e dor do paciente, a angústia explícita dos familiares, a angústia latente da equipe, e a angústia negada dos médicos. Nesse sentido, a Psicologia Hospitalar trabalha com tais aspectos psicológicos, considerando cada sujeito individualmente, e buscando auxiliar a relação entre os componentes da tríade paciente-família-equipe de saúde (Simonetti, 2004).

No atual cenário mundial, emerge um novo desafio para o atendimento psicológico no contexto hospitalar e da clínica psicológica. Dessa forma, a pandemia do coronavírus evidenciou não só as preocupações com a saúde física da população, como também o sofrimento psicológico que tem afligido a população geral e os profissionais de saúde que atuam na linha de frente. Um evento como esse leva a impactos psicológicos e sociais que abalam a capacidade de enfrentamento da sociedade. Por isso, ações emergenciais se tornam necessárias e mobilizam diversas áreas do conhecimento, incluindo a psicologia, para sugerir formas de enfrentar a crise (Schmidt, Crepaldi, Bolze, Neiva-Silva \& Demenech,(2020); Faro, Nakano, Reis, Silva \& Vitti, 2020).

Com as novas necessidades observadas, o Conselho Federal de Psicologia (CFP, 2020) vem trazendo novas resoluções a respeito do atendimento on-line, para que os profissionais da psicologia possam realizar esse tipo de atendimento e resguardarse frente a políticas de prestação de serviços virtuais e contribuir para políticas de prevenção, tanto para o âmbito hospitalar, como para o âmbito de saúde mental e na clínica psicológica. Além disso, o trabalho na linha de frente do combate à pandemia pode motivar ou intensificar a depressão, ansiedade e estresse, devido ao aumento da carga de trabalho e às mudanças frequentes nos protocolos de atendimento, em decorrência de novas descobertas sobre o coronavírus (Schmidt et al., 2020). 
Dessa forma, o objetivo deste estudo é verificar quais os impactos que a pandemia trouxe para a saúde mental e capacidade laboral de profissionais de psicologia no contexto hospitalar e da saúde na clínica psicológica. Tal investigação surgiu da constatação da escassez de literatura acerca da saúde de psicólogos frente ao contexto de pandemia e do desejo de analisar possíveis repercussões na saúde destes profissionais, bem como as dificuldades e sofrimentos psíquicos decorrentes.

\section{Metodologia}

A proposta foi selecionar artigos científicos que contemplam observações sobre os impactos que o psicólogo em contexto de saúde clínica e hospitalar vem sofrendo no atual momento da pandemia do coronavírus. Optou-se pela realização da revisão para que esta possa subsidiar futuros estudos na área.

Para este, realizou-se um estudo de revisão integrativa de literatura. A revisão integrativa foi escolhida como recurso metodológico, porque permite sumarizar estudos realizados anteriormente na temática selecionada. Assim, é permitido colocar em discussão os métodos, fontes, objetivos e resultados, o que permite estabelecer conclusões em relação ao campo de conhecimento demarcado (Botelho, Cunha \& Macedo, 2011). A coleta de dados foi realizada em fevereiro de 2021. Para isso, foram pesquisados os artigos indexados com os seguintes descritores: "saúde mental", "pandemia", "atenção terciária em saúde", "profissionais da saúde" associados ao operador booleano "AND". Esses, por sua vez, foram escolhidos de acordo com o Descritores em Ciências da Saúde - DeCS.

As buscas foram realizadas na na plataforma do Google Scholar, no periódico da Coordenação de Aperfeiçoamento de Pessoal de Nível Superior (CAPES), na Scientific Eletronic Librabry Online (SCIELO) e nas bases de dados da Literatura LatinoAmericano e do Caribe em Ciências da Saúde (LILACS) e no Portal de Periódicos Eletrônicos de Psicologia (PePSIC) inserido na Biblioteca Virtual em Saúde (BVS), bem como selecionando artigos disponíveis nas bases de dados de Literatura Latino Americana em Ciências da Saúde (LILACS). A estratégia de busca dos estudos, baseou-se na presença dos termos no título do trabalho, nas palavras-chave e/ou no resumo.

O presente estudo tem como critérios de inclusão: (1) estudos divulgados em formato de artigos científicos, disponibilizados completos na íntegra; (2) estudos publicados nos idiomas português, inglês e espanhol; (3) estudos que abordaram a atuação de psicólogos em contexto clínico e hospitalar/atenção terciária. Como critérios de exclusão foram estabelecidos: (1) apresentação sob formato de livro ou capítulo de livro, dissertação, tese, editorial, comentário, crítica, resenha, revisões, anais e outros relatórios científicos; (2) estudos que focalizavam na atuação de outros profissionais de saúde que não o psicólogo; (3) artigos não revisados por pares e (4) e estudos que reportaram a atuação profissional dos psicólogos em outros níveis de atenção. A figura 1 demonstra o quantitativo de artigos encontrados na presente busca.

Após todo o processo de seleção, os quatro artigos selecionados foram lidos na íntegra e, a partir da leitura, realizou-se a caracterização da produção encontrada. Organizaram-se os achados com base nos seguintes dados: I - Título dos artigos; II Nome dos Autores e Ano do Estudo. 
Figura 1 - Quantidade de artigos encontrados e selecionados, de acordo com critérios de seleção (inclusão e exclusão), 2021.

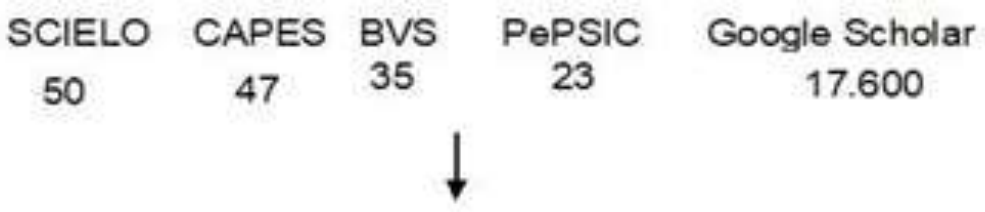

\subsection{5 artigos encontrados nas bases de dados}

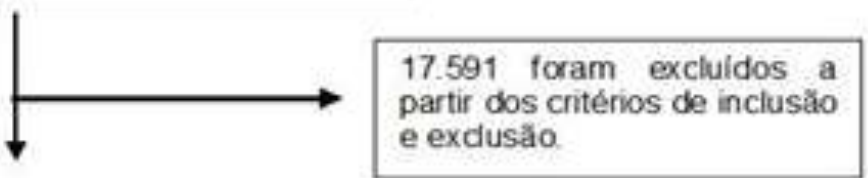

$\begin{array}{ccccc}\text { SCIELO } & \text { CAPES } & \text { BVS } & \text { PePSIC } & \text { Google Scholar } \\ 01 & 04 & 08 & 01 & 01\end{array}$

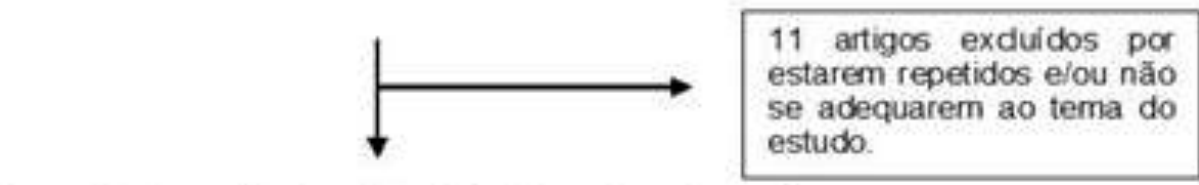

4 artigos selecionados e lidos integ almente.

Fonte: Autores (2021).

\section{Resultados}

Resultante da proposta de revisão integrativa de literatura acerca das possibilidades dos impactos ao profissional de psicologia no vivenciamento da mais recente pandemia do coronavírus, questões foram evidenciadas nos quatro artigos publicados em 2020 e que são discutidos a seguir.

Quadro 1 - Título dos artigos, autores e ano de publicação.

\begin{tabular}{|l|l|}
\hline Título & Autor (es) e ano de publicação \\
\hline $\begin{array}{l}\text { A psicologia Hospitalar e da Saúde no enfrentamento do coronavírus: } \\
\text { necessidade e proposta de atuação }\end{array}$ & Grincenko, F. R. S. (2020) \\
\hline $\begin{array}{l}\text { A saúde dos profissionais de saúde no enfrentamento da pandemia de } \\
\text { covid-19 }\end{array}$ & $\begin{array}{l}\text { Teixeira, C .F .S., Soares, C. M., Souza, E. A,. Lisboa, E. S., } \\
\text { Pinto, L. C. M., Andrade, L. R., Espiridão, M. A. (2020) }\end{array}$ \\
\hline Atuação do psicólogo na saúde mental da população diante da pandemia & Danzmann, P. S., Silva, A. C. P., Guazina, F. M. N. (2020) \\
\hline $\begin{array}{l}\text { Impacto da pandemia na saúde mental de profissionais de saúde que } \\
\text { trabalham na linha de frente da COVID-19 e o papel da psicoterapia }\end{array}$ & $\begin{array}{l}\text { De morais, C. P. T., Gomes, G. M. B., Machado, L. C. S., } \\
\text { Daumas, L. P., Gomes, M. M. B. (2020) }\end{array}$ \\
\hline
\end{tabular}

Fonte: Autores (2021).

Em relação ao coronavírus, um estudo apontou para a exposição direta às possibilidades de contágio ao vírus, ao profissional que trabalha diariamente no atendimento de pacientes e profissionais da saúde que estão atuantes neste meio. Também pôde-se observar dados em relação a escassez de materiais de proteção individual, contato constante e iminente com o sofrimento, a dor e a morte, fatores que podem levar ao desenvolvimento de quadros de ansiedade, depressão, síndrome de burnout, transtorno de estresse pós-traumático, transtornos psicossomáticos e o uso de substâncias como álcool e outras drogas, além de remédios sem indicação (Grincenkov, 2020). 
Esses apontamentos também são corroborados com os achados de outra investigação que demonstrou que o risco de contaminação pelo vírus vem resultando em processos de afastamento do trabalho, intenso sofrimento psíquico, transtornos ansiosos, problemas no sono, medo de adoecer e de contribuir para o contágio (Teixeira et al., 2020). Salienta-se que essas são as variáveis mais prevalentes no contexto laboral, desse período pandêmico, enfrentados pelos profissionais de psicologia junto a equipe multidisciplinar. Além disso, os psicólogos estão vivenciando desafios devido a sobrecarga frente ao substancial aumento da demanda referente aos efeitos negativos do coronavírus na saúde mental da população em geral, de modo a destacar os profissionais da saúde (De Morais et al., 2020)

Frente a esse contexto, estão sendo oferecidas medidas alternativas para o atendimento da população. Os psicólogos adaptaram-se para o atendimento psicológico clínico on-line, disponibilizando-se a realizar intervenções remotas, trabalho que pode amenizar ou prevenir consequências psicológicas eliciadas por esta nova realidade, como distúrbios psiquiátricos ou do sono. Este novo formato de trabalho é considerado uma forma eficiente de evitar o contágio do vírus e conta com possibilidades de adaptar-se à rotina dos profissionais de saúde e, dessa forma, atender as demandas da população que busca cuidados (De Morais et al., 2020).

Mesmo com o atendimento psicológico on-line sendo considerado uma estratégia viável, as diversas resistências que esse meio sofre são expressadas. Nessa perspectiva, ainda são evidenciadas críticas e resistências por parte dos usuários que não conseguem aderir à forma de atendimento on-line. Um dos motivos seria a não credibilidade na implementação da aliança terapêutica solidificada e segura, ou seja, o vínculo entre psicólogo e paciente. Outro motivo está alinhado ao risco da não confidencialidade e sigilo das informações, o que pode intimidar o cliente ao verbalizar seus sentimentos, pensamentos e sensações. Neste sentido, encontram-se reais dificuldades acompanhamento virtual, mas reforça-se que o atendimento clínico on-line mostra como a psicologia se adaptou para não descuidar da saúde mental da sociedade (Danzmann, Silva \& Guazina, 2020).

Adicionalmente, ressalta-se que não só o atendimento clínico vem sofrendo alterações e impactos decorrentes da pandemia. Alguns estudos, depararam-se com as dificuldades que o psicólogo inserido no âmbito hospitalar vem vivenciando e constataram que ser psicólogo neste contexto acaba por ser um desafio, porque a formação em psicologia ainda é deficitária. Dessa forma, seria necessário a criação de protocolos de intervenção psicológica para se adaptar ao formato remoto a fim de possibilitar a melhora da saúde mental, suporte à estabilidade do humor e do sono, diminuição do estresse e acolhimento a quem recorre a este auxílio (Danzmann, Silva \& Guazina, 2020; Grincenkov, 2020).

\section{Discussão}

O principal objetivo desse estudo foi rastrear, na literatura, por meio de uma revisão integrativa, quais os impactos que a pandemia trouxe para a saúde mental e capacidade laboral de profissionais de psicologia no contexto hospitalar e da saúde clínica. Essa reflexão se faz necessária uma vez que há discussões voltadas para a saúde mental de outros profissionais atuantes na linha de frente do Coronavírus (e.g.médicos e enfermeiros).

Nessa perspectiva, evidenciou-se que em ambos os contextos estudados a eclosão de sentimentos de medo frente ao incerto, à gravidade de situação, possibilidade de agravo pessoal, questões acerca do futuro da atuação profissional, ansiedade e possibilidade de vivenciar quadros de estresse no trabalho e burnout foram presentes (Grincenkov, 2020; Teixeira et al., 2020; Danzmann; Silva; Guazina, 2020). Em momento que precedeu a idealização do tema de pesquisa, os autores proponentes do manuscrito, questionaram-se sobre a possibilidade de existir relatos científicos acerca da saúde do profissional que enfrenta direta e indiretamente a pandemia do coronavírus.

Nesse sentido, discutiu-se sobre a iminência do dano psicológico e físico do profissional que está inserido em contexto clínico e em âmbito hospitalar de atenção terciária a saúde, além de temas relacionados a acessibilidade aos equipamentos 
necessários para a realização do serviço proposto, dificuldades acerca do relacionamento com a equipe multiprofissional de saúde, dificuldade quanto às limitações impostas direta e indiretamente ao contato com o familiar e dificuldades quanto à prestação do trabalho em si, com limitações de base quanto como proceder ao atendimento de crise.

Desta forma, e após as análises que foram julgadas como necessárias e pertinentes, confirmou-se os objetivos definidos em momento anterior à pesquisa, corroborada pela literatura estudada, que apontaram preocupações semelhantes. Os artigos remeteram de forma objetiva as incertezas e impactos vividos dentro da prestação do serviço de saúde e os danos que já se fazem presentes dentro do quadro de profissionais. Quanto a esse fato exposto, apesar da literatura ainda carecer de tempo para desenvolver-se, já convergem entre si, sobre os infortúnios vivenciados pelos profissionais de saúde (Grincenkov, 2020; Teixeira et al., 2020; Danzmann; Silva; Guazina, 2020).

Quanto a possibilidade de escassez de materiais de trabalho e as consequências práticas da ausência deles, os achados levantados apontam para a realidade vivida dentro de hospitais do país, em que retratou a real falta e as melhores condições, à escassez do que se julga de estrita necessidade para a realização de um trabalho seguro ao paciente, e de segurança ao profissional. Também foi questionado sobre o quão imbricado o sofrimento quanto ao trabalho prestado, pode estar atrelado com a baixa perspectiva de municiamento de instrumento de trabalho (Grincenkov, 2020; Teixeira et al., 2020)

No que tangencia o âmbito de relacionamento entre os profissionais e levando em consideração a possibilidade de desencontros entre amplitudes práticas e teóricas é levantado um ponto interessante sobre a falta de preparo de base ao profissional de psicologia, em específico. Devido ao fluxo de pacientes e da emergência, o contato e atenção, itens de extrema importância dentro do atendimento psicológico, fica limitado e, consequentemente, o trabalho sofre interferências (Andrade \& Espiridião, 2020).

Nesse sentido, cabe ao profissional da psicologia a desenvoltura de adaptar algo que está estabelecido há muito tempo, a fim de prestar o melhor serviço possível, dado as circunstâncias e novos costumes em relação à tecnologia. Por isso, começa a surgir por conta das necessidades de distanciamento social e emergencial, alternativas que vão de encontro com a digitalização do mundo. Ademais, sendo um dos últimos a sentirem essa necessidade, o atuante de psicologia se vê criando um mundo digital de possibilidade de atendimento, prestação de ajuda e de cuidado (Morais et al., 2020).

Por decorrência dos planos de diminuição de contágio, os psicólogos, frequentemente, acabam por se verem ilhados e impedidos do contato pessoal com seus clientes e pacientes. Há deslocamento de esforço ao criar um método de atendimento em clínica psicológica que seja passível de adaptabilidade e efetividade enquanto existe a necessidade de entendimento de funcionalidade terapêutica por parte da população. Disto, discute-se sobre os impactos sofridos por estes profissionais, ao se verem desarmados de noção terapêutica atrelada a concepção de que uma terapia efetiva seria uma terapia olho no olho, enquanto na realidade, qualquer esforço em busca da melhora psíquica, se faz promissora e efetiva (Grincenkov, 2020).

Discute-se por meio desta revisão, e evidencia-se com a mesma, a necessidade do cuidado a quem cuida e assistência aos que necessitam dela, bem como novos protocolos para a melhora do trabalho do psicólogo em âmbito de saúde clínica e hospitalar. Em meio a um cenário de caos e colapso da saúde, cabe dedicar atenção para aquele que segura toda essa responsabilidade e que muitas vezes negligencia-se em prol do próximo. Mesmo com pouco tempo desde a eclosão da pandemia, os ínfimos artigos já convergem entre si e alinham-se com a perspectiva daqueles que observam de fora.

\section{Considerações Finais}

Este estudo surgiu da percepção de que os profissionais da psicologia, na condição de graduandos ou formados, estão inseridos no espaço de detenção e prevenção dos danos do coronavírus, mas estes ainda não ocupam a devida atenção da ciência. Neste momento, pode-se discutir sobre o profissional da saúde, em especial, da psicologia, inserido no meio pandêmico hospitalar 
e clínico, em qual a ênfase é ressaltar a qualidade e eficácia desse profissional e compreender quais os impactos que este sofreu e ainda sofre neste momento.

É perceptível a importância de que todos os profissionais da saúde ganharam destaque em 2020, considerado o ano pandêmico. Em meio a eclosão de casos, óbitos e caos decorrente da pandemia, a saúde destes profissionais pode ser agravada e comprometida, de modo a surtir a necessidade do olhar referente ao estresse, ansiedade, problemas traumáticos e sociais, além de novas adaptações para que o trabalho fosse feito. Cabe ressaltar que o psicólogo é um profissional de relevância na saúde em geral, e principalmente, mental. Estes profissionais também se encontram em total vulnerabilidade em ambientes hospitalares e ambiente clínico, seja frente ao contágio do vírus, ou frente à demanda decorrente deste. Por isso, reforça-se que o olhar também deve se direcionar a essa categoria para que assim o cuidado efetivo e humanizado para os outros possa ser assegurado com qualidade.

Esse estudo demonstra potencialidades ao reportar um olhar de cuidado aos profissionais de psicologia e da sua relevância no enfrentamento a covid-19, no entanto, reconhecem-se suas limitações. Notou-se que poucos artigos de referência foram encontrados, o que infere que essa demanda de saúde pública é recente. Por esse motivo, cabe ressaltar que as discussões sobre profissionais da saúde e pandemia ganham ênfase nos estudos em contextos gerais e não específicos como este. Além disso, o campo de estudo enfocou para a atuação primária (clínica) e terciária (hospitalar), o que faz sugerir que futuros estudos poderão ampliar essa perspectiva sobre esse impacto, por exemplo, no âmbito escolar/educacional organizacional e do trabalho ou em outros níveis de atenção (e.g. primário e secundário). Por fim, uma agenda de pesquisa sugerida seria uma investigação de delineamento quantitativo e qualitativo que vise compreender dados no que está relacionada a sintomas ansiosos, depressivos e adaptações no meio de trabalho pandêmico.

\section{Financiamento}

Para a condução desse estudo não houve financiamento por agências de fomento, sendo de inteira responsabilidade dos autores todos os custos oriundos para a construção deste manuscrito.

\section{Conflitos de Interesse}

Os autores declaram que não existem interesses concorrentes.

\section{Referências}

Angerami-Camon, V. A. (2001). Psicologia Hospitalar: teoria e prática. São Paulo: Cengage Learning

Brasil. Ministério da Saúde. 2020. http://www.saude.gov.br/. Acesso em: 21 fev. 2021.

Botelho, L. de L.R.; Cunha, C. C.de A.; \& Macedo, M. O método da revisão integrativa nos estudos organizacionais. (2011). Gestão e Sociedade, v. 5, n. 11, p. 121-136.

Conselho Federal de Psicologia (Brasil). (2020). Regulamentação de serviços psicológicos prestados por meio de Tecnologia da Informação e da Comunicação durante a pandemia do COVID-19. Conselho Federal de Psicologia. 2. ed., Brasília: CFP.

Danzmann, P.S.; Silva, A. C. P. da; G., \& Félix, M. N. (2020). Atuação do psicólogo na saúde mental da população diante da pandemia. J. nurs. health, p. $20104015-20104015$.

Faro, A., Bahiano, M. A., Nakano, T. C., Reis, C., Silva, B. F. P., \& Vitti, L. S. (2020). COVID-19 e saúde mental: a emergência do cuidado. Estudos de Psicologia (Campinas), 37, e200074. https://doi.org/10.1590/1982-0275202037e200074

Grincenkov, Fabiane Rossi. (2020). A Psicologia Hospitalar e da Saúde no enfrentamento do coronavírus: necessidade e proposta de atuação. Hu Revista, v. 46, p. 1-2.

Moraes, C. P., Gomes, G. M. B., Machado, L. C. S., Daumas, L. P. \& Gomes, M. M. B. (2021). Impacto da pandemia na saúde mental dos profissionais de saúde que trabalham na linha de frente da Covid-19 e o papel da psicoterapia. Brazilian Journal of Development, v. 7, n. 1, p. 1660-1668.

Prado, A. D., Peixoto, B. C., Silva, A. M. B. e Scalia, L. A. M. (2020). A saúde mental dos profissionais de saúde frente à pandemia do COVID-19: uma revisão integrativa. Revista Eletrônica Acervo Saúde, n. 46, p. e4128-e4128. 
Research, Society and Development, v. 10, n. 4, e33610414140, 2021

(CC BY 4.0) | ISSN 2525-3409 | DOI: http://dx.doi.org/10.33448/rsd-v10i4.14140

Schmidt, B., Crepaldi, M. A., Bolze, S. D. A., Neiva-Silva, L., \& Demenech, L. M. (2020). Saúde mental e intervenções psicológicas diante da pandemia do novo coronavírus (COVID-19). Estudos de Psicologia (Campinas), 37, e200063. Doi: http://dx.doi.org/10.1590/1982-0 275202037e200063

Simonetti, A. (2004). Manual de Psicologia Hospitalar: O Mapa da Doença. São Paulo: Casa do Psicólogo.

Teixeira, C. F. S, Soares, C. M., Souza, E. A., Lisboa, E. S., Pinto, I. C. M., Andrade, L. R. e Espiridião, M. A. (2020). A saúde dos profissionais de saúde no enfrentamento da pandemia de Covid-19. Ciência \& Saúde Coletiva, v. 25, p. 3465-3474. 